\title{
Cage decay, near constant loss, and crossover to cooperative ion motion in ionic conductors: Insight from experimental data
}

\author{
K. L. Ngai \\ Naval Research Laboratory, Washington, DC 20375-5320 \\ C. León \\ GFMC, Departamento Fisica Aplicada III, Facultad de Ciencias Fisicas, Universidad Complutense de Madrid, \\ Avda. Complutense s/n, 28040 Madrid, Spain
}

(Received 29 April 2002; published 30 August 2002)

\begin{abstract}
Experimental frequency-dependent conductivity relaxation spectra of a number of molten, glassy, and crystalline ionic conductors that show both the presence of the near constant loss (NCL) and the cooperative ion hopping contribution are analyzed. On decreasing frequency, the NCL appears first but terminates at some frequency $\nu_{x 1}$. At still a lower frequency $\nu_{x 2}$ the cooperative ion hopping dispersion takes over. The independent ion hopping frequency $\nu_{0}$ of the coupling model is calculated from the parameters characterizing the cooperative ion hopping dispersion. It is found for all ionic conductors that $\nu_{x 1} \gg \nu_{0}$, and $\nu_{0}$ always fall inside the frequency region $\nu_{x 1}>\nu>\nu_{x 2}$. The empirical results leads to a qualitative theory for the origin of the NCL, which gives physical meanings of the two crossover frequencies $\nu_{x 1}$ and $\nu_{x 2}$, as well as explaining the role of the independent hopping frequency $\nu_{0}$, in determining them. The weak temperature dependence of the NCL has been recaptured by the qualitative theory. An improved understanding is gained of the evolution of the ion dynamics from early times when the cages decay very slowly with time, giving rise to the near constant loss, to long times when ions move cooperatively, leading finally to dc conductivity.
\end{abstract}

DOI: 10.1103/PhysRevB.66.064308

PACS number(s): 66.30.Hs, 66.30.Dn, 77.22.Gm

\section{INTRODUCTION}

One of the frontiers of research in the field of dynamics of ions in ionic conductors is the origin of the ubiquitous near constant loss (NCL)

$$
\varepsilon_{\mathrm{NCL}}^{\prime \prime}(\omega) \approx A \omega^{-\alpha},
$$

where $\alpha$ is nearly zero and $A$ is a constant with weak temperature dependence that is well described by $\exp \left(T / T_{0}\right)$, where $T_{0}$ is a temperature that is usually larger than $T$. The NCL corresponds to an almost linear frequency-dependent term

$$
\sigma_{\mathrm{NCL}}^{\prime}(\omega)=\omega \varepsilon_{\mathrm{NCL}}^{\prime \prime}(\omega) \approx A \omega^{1-\alpha}
$$

in the real part of the complex conductivity. The existence of NCL in glassy ionic conductors is well known. Evidences for its existence was suggested repeatedly over the span of several decades ${ }^{1-12}$ and it is now considered to be a universal characteristic of ionic conductors, ${ }^{13,14}$ although till now there are only a few investigations of its properties. ${ }^{11-19}$ This NCL contribution appears at higher frequency than the ion hopping ac conductivity $\sigma_{\text {hop }}^{\prime}(\omega)$. The latter is assumed by some workers to be represented by the Jonscher expression ${ }^{20}$

$$
\sigma_{J}^{*}(\omega)=\sigma^{\prime}(\omega)+i \sigma^{\prime \prime}(\omega)=\sigma_{0}\left[1+\left(i \omega / \omega_{p}\right)^{n}\right],
$$

where $n_{J}$ is a fractional exponent, $\sigma_{0}$ is the de conductivity, and $\omega_{p}$ a characteristic relaxation frequency. Alternatively, ${ }^{21}$ the ion hopping ac conductivity is also well described by the one-sided Fourier transform

$$
M^{*}(\omega)=M^{\prime}+i M^{\prime \prime}=M_{\infty}\left[1-\int_{0}^{\infty} d t \exp (-i \omega t)(-d \Phi / d t)\right]
$$

of the Kohlrausch stretched exponential function

$$
\Phi(t)=\exp \left[-(t / \tau)^{1-n}\right] .
$$

The ion hopping contribution $\sigma_{n}^{\prime}(\omega)$ obtained from Eqs. (4) and (5) together with the Maxwell relation $\sigma_{n}^{\prime}(\omega)$ $=\operatorname{Re}\left[i \omega \varepsilon_{0} / M^{*}(\omega)\right]$, is similar to the Jonscher's expression in having the dc conductivity $\sigma_{0}$ at low frequencies, and it increases as a power law $(\omega \tau)^{n}$ at high frequencies instead of $\left(\omega / \omega_{p}\right)^{n_{J}}$ from the Jonscher expression. The advantage of using Eqs. (4) and (5) over the Jonscher expression to describe experimental data was pointed out by Moynihan. ${ }^{21}$ Both $\sigma_{0}$ and the electrical conductivity relaxation time $\tau$ in Eq. (5) have the same temperature dependence. Experimental evidences indicate that the stretched exponential time dependence of electrical relaxation is caused by ion-ion correlation, and therefore $\sigma_{n}^{\prime}(\omega)$ is a cooperative ion hopping contribution.

The properties of the NCL differ ${ }^{14}$ in many respects from the ion hopping transport contribution and can be considered to have different physical origins. For example, while $\sigma_{\mathrm{NCL}}^{\prime}(\omega)$ has a weak temperature dependence, either $\sigma_{J}^{*}(\omega)$ or $\sigma_{n}^{\prime}(\omega)$ has strong thermally activated temperature dependence. From the properties of the NCL, it was concluded that nevertheless it comes from the ions. ${ }^{14}$ The evidences include the observed approximately linear increase of the NCL with concentration of the mobile ions, ${ }^{6,16}$ the very different effect when mixing different alkalis, ${ }^{8,9,14}$ and the existence of the NCL in crystalline ionic conductors. ${ }^{22-24}$ The last experi- 
mental fact rules out the possibility that the NCL of present interest originates from the glassy matrix in which the ions are imbedded.

At sufficiently low temperature under isofrequency condition or high frequencies under isothermal condition, the NCL is the only contribution that is seen experimentally. In a recent paper $^{18}$ an analysis of the constant loss contribution to the ac conductivity, in the frequency range $10 \mathrm{~Hz}-1 \mathrm{MHz}$ and finely spaced temperatures down to $8 \mathrm{~K}$, was reported for two different $\mathrm{Li}$ ionic conductors, one crystalline $\left(\mathrm{Li}_{0.18} \mathrm{La}_{0.61} \mathrm{TiO}_{3}\right)$ and the other glassy $\left(61 \mathrm{SiO}_{2} \cdot 35 \mathrm{Li}_{2} \mathrm{O}\right.$ $\cdot 3 \mathrm{Al}_{2} \mathrm{O}_{3} \cdot 1 \mathrm{P}_{2} \mathrm{O}_{5}$ ). At lower temperatures, a NCL corresponding to near linear frequency-dependent ac conductivity is the observed contribution. As temperature is increased a crossover from the near constant loss to a fractional powerlaw frequency dependence of the ac conductivity [Eq. (3) or Eqs. (4) and (5)] is observed. At any fixed frequency $\omega$, this crossover occurs at a temperature $T$ determined approximately by the relation

$$
\omega \approx \omega_{\infty} \exp \left(-E_{m} / k T\right)
$$

The activation energy $E_{m}$ turns out to be significantly smaller than the dc conductivity activation energy $E_{\sigma}$. From this observed property it has been shown ${ }^{25}$ that the NCL term $A \omega$ is not a contribution to $\sigma^{\prime}(\omega)$ that can be added on top of the ion hopping ac conductivity term represented by the Jonscher expression as is commonly believed, or by $\sigma^{\prime}(\omega)$ obtained from Eqs. (4) and (5) of the electric modulus. Had these two contributions been additive contributions, the crossover frequency would have $E_{\sigma}$ as the activation energy instead of the significantly smaller observed $E_{m}$. Thus, excluding the vibrational and boson peak contributions, in the high frequency regime there is only the NCL. On decreasing frequency (or increasing time) the NCL will no longer holds at some point $\omega_{x 1}$, afterwards $\sigma^{\prime}(\omega)$ undergoes a transition over some frequency range with continuously decreasing slope and finally, starting at $\omega_{x 2}$, assumes the frequency dependence of $\sigma_{\text {hop }}^{\prime}(\omega)$ given by either $\sigma_{J}^{\prime}(\omega)$ or $\sigma_{n}^{\prime}(\omega)$ that have fractional power laws $\left(\omega / \omega_{p}\right)^{n_{J}}$ or $(\omega \tau)^{n}$ at their high frequency ends.

In the sections to follow, we shall characterize the crossover frequencies $\omega_{x 1} \equiv 2 \pi \nu_{x 1}$ and $\omega_{x 2} \equiv 2 \pi \nu_{x 2}$ from isothermal $\sigma^{\prime}(\omega)$ data of glassy and molten ionic conductors. We include all data we know of that have been obtained over a very broad frequency range to exhibit at the same temperature both the NCL and the $\sigma_{\text {hop }}^{\prime}(\omega)$. This kind of data enables unambiguous determination of $\nu_{x 1}$ and $\nu_{x 2}$ at the same temperature. When compared with the independent ion hopping relaxation frequency $\nu_{0} \equiv 1 /\left(2 \pi \tau_{0}\right)$, where $\tau_{0}$ is the familiar independent ion hopping relaxation time of the coupling model, ${ }^{26-28}$ it is found that $\nu_{0}$ lies in between $\nu_{x 1}$ and $\nu_{x 2}$. This general result leads to a proposal for the origin of the NCL, an interpretation of the crossover frequencies $\nu_{x 1}$ and $\nu_{x 2}$ and an explanation of the weak temperature dependence of the NCL.

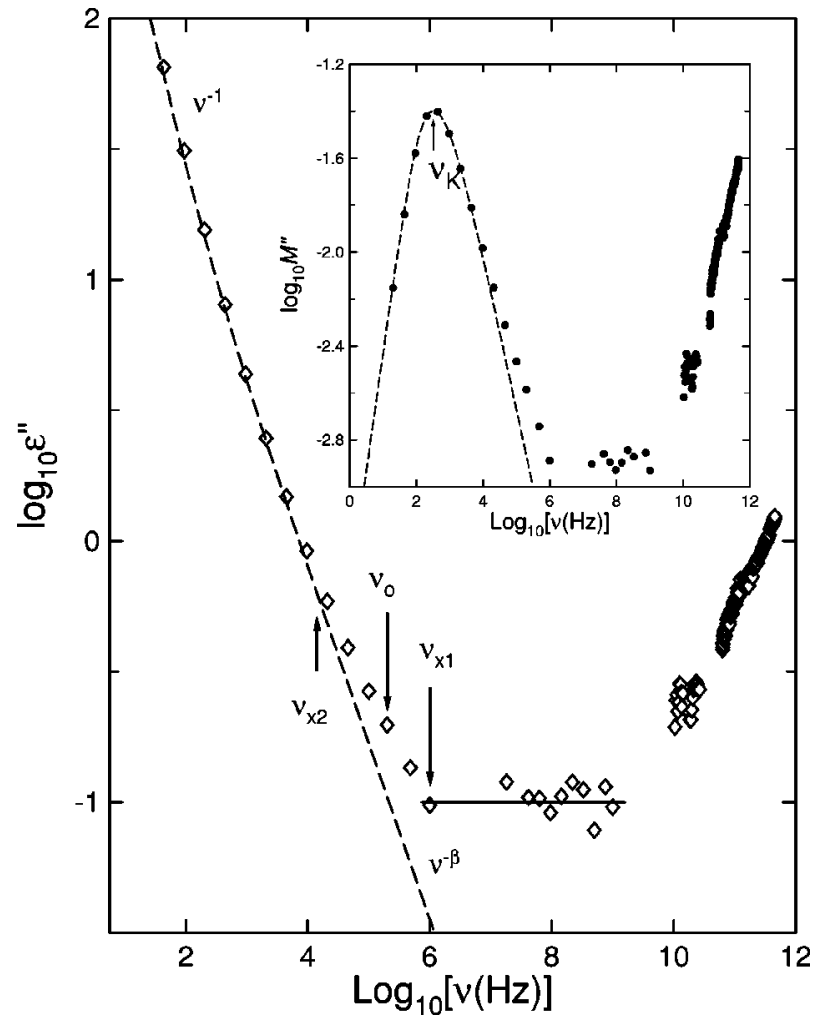

FIG. 1. Dielectric loss as a function of frequency of CKN at $T$ $=342 \mathrm{~K}$ showing the existence of the NCL over 3 decades in frequency. The horizontal dashed line indicates the NCL. The other dashed line is the fully cooperative ion conductivity relaxation calculated from the Kohlrausch fit to the electric loss modulus $M^{\prime \prime}$ data shown in the inset as the dashed line with $\beta=0.66$. The deviation of the data from the Kohlrausch fit at higher frequencies is marked by one crossover frequency, $\nu_{x 2}$. The deviation of the data from the NCL at lower frequencies is marked by the other crossover frequency $\nu_{x 1}$. The location of the independent relaxation frequency of the $\mathrm{CM} \nu_{0} \equiv 1 / 2 \pi \tau_{0}$, is also indicated. $\tau_{0}$ is the independent relaxation time calculated from the CM. Data after Refs. 39 and 40.

\section{THE CROSSOVER FREQUENCIES $\nu_{x 1}$ AND $\nu_{x 2}$ FROM EXPERIMENTAL DATA}

\section{A. $0.4 \mathrm{Ca}(\mathrm{NO3})_{2}-0.6 \mathrm{KNO}_{3}(\mathrm{CKN})$}

Extensive electrical relaxation data of CKN over unusually broad frequency and temperature ranges were obtained by Lunkenheimer et al. ${ }^{29,30}$ In Fig. 1, we show first their $\varepsilon^{\prime \prime}$ data at $342 \mathrm{~K}$ as a function of frequency $\nu$, which is related to the angular frequency $\omega$ by $\omega=2 \pi \nu$. The data shown by points indicate the existence of a NCL that extends over about three decades and there is a cross over to a power law $\varepsilon^{\prime \prime}(\nu) \propto \nu^{-\beta}$ at lower frequencies, with $\beta=1-n$, and eventually $\varepsilon^{\prime \prime} \propto \nu^{-1}$ at even lower frequencies in the dc conductivity regime, as illustrated in the figure. The inset of the same figure shows the same data but as $M^{\prime \prime}(\nu)$. The dashed line is the fit by Eqs. (4) and (5) to the data with $\beta \equiv(1$ $-n)=0.66$ and $\tau=6.6 \times 10^{-4} \mathrm{~s}$. The data in the $M^{\prime \prime}$ representation shows also the crossover from NCL to the power law $M^{\prime \prime} \propto \nu^{-\beta}$. Although the crossover from NCL to $\nu^{-\beta}$ is broad for both $\varepsilon^{\prime \prime}$ and $M^{\prime \prime}$, it can be observed that the rise from the flat loss occurs nonetheless quite abruptly, as found 


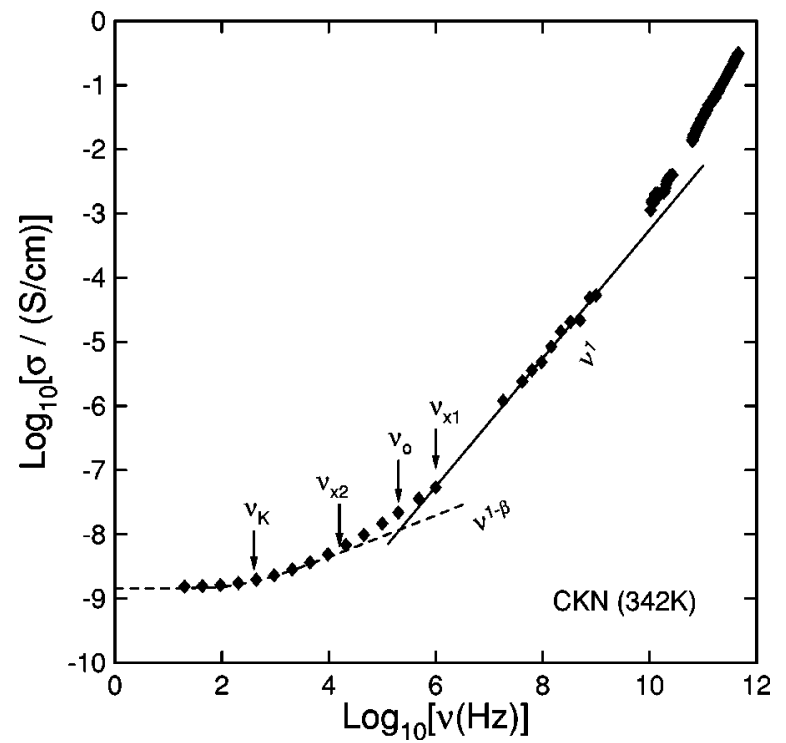

FIG. 2. Replotting the CKN data of Fig. 1 as $\log \sigma^{\prime}(\nu)$ versus $\log ($ frequency $/ \mathrm{Hz})$ to show the near linear frequency dependence of the NCL in Fig. 1. The dashed line that includes the dc conductivity and the high frequency power law $\nu^{1-\beta}$ or $\nu^{n}$ corresponds to the Kohlrausch fit to the electric modulus shown as the dashed line in the inset of Fig. 1 with $n=0.34$. The dashed line in the main figure is the fully cooperative ion conductivity relaxation.

previously for $\mathrm{Li}_{0.18} \mathrm{La}_{0.61} \mathrm{TiO}_{3}$ (LLTO) ${ }^{18}$ It is worthwhile to point out that the dashed line in the main part of Fig. 1 having $\nu^{-1}$ at low frequencies and $\nu^{-\beta}$ at higher frequencies is actually calculated from the fit to the electric modulus by the identity $\varepsilon^{*} \equiv\left(1 / M^{*}\right)$. The other dashed line in the main figure is $\varepsilon_{\beta}^{\prime \prime}(\nu)$ calculated from the identity $\varepsilon_{\beta}^{\prime \prime}(\nu)$ $=\operatorname{Im}\left\{1 / M_{\beta}^{*}\right\}$ and it is the loss from cooperative hopping of ions. The $\varepsilon^{\prime \prime}$ data indicate the existence of a NCL that extends over about three decades and there is a crossover to a power law $\nu^{-\beta}$ at lower frequencies and eventually $\varepsilon^{\prime \prime}$ $\propto \nu^{-1}$ at even lower frequencies in the dc conductivity regime. The data in the $M^{\prime \prime}$ representation shows also the crossover from NCL to the power law $M^{\prime \prime} \propto \nu^{-\beta}$. Some readers may be more familiar with the NCL having the near $\nu^{1}$ dependence in the $\sigma^{\prime}(\nu)$ representation of the data as shown in Fig. 2. Again the dashed curve that tends to the dc conductivity at low frequencies and the power law $\nu^{(1-\beta)}$ at high frequencies is $\sigma_{\beta}^{\prime}(\nu)$ calculated from the identity $\sigma_{\beta}^{\prime}(\nu) \equiv \omega \varepsilon_{\beta}^{\prime \prime}(\nu)$ and again it comes from cooperative ion hopping. The NCL terminates at lower frequencies quite distinctly at $\nu_{x 1}$. It can be seen by inspection of Fig. 1 through the rapid rise of $\varepsilon^{\prime \prime}(\nu)$ from the flat loss at frequencies below $\nu_{x 1}$, as found previously in $\mathrm{Li}_{0.18} \mathrm{La}_{0.61} \mathrm{TiO}_{3}$ (LLTO). ${ }^{18} \mathrm{On}$ further lowering the frequency, starting at $\nu_{x 2}, \varepsilon^{\prime \prime}$ and $M^{\prime \prime}$ assume the $\nu^{-\beta}$ dependence and $\sigma^{\prime}(\nu)$ assumes the $\nu^{1-\beta}$ dependence of the cooperative ion hopping. Thus the crossover of the $\varepsilon^{\prime \prime}(\nu)$ data from NCL to $\varepsilon_{\beta}^{\prime \prime}(\nu)$ [or $\sigma(\nu)$ data from NCL to $\left.\sigma_{\beta}^{\prime}(\nu)\right]$ is not sharp, occurring over the frequency range $\nu_{x 1}>\nu>\nu_{x 2}$. The values of $\nu_{x 1}$ and $\nu_{x 2}$ depend on the criterion of when the crossover has occurred. We use a $5 \%$ deviation as the criterion whenever there are

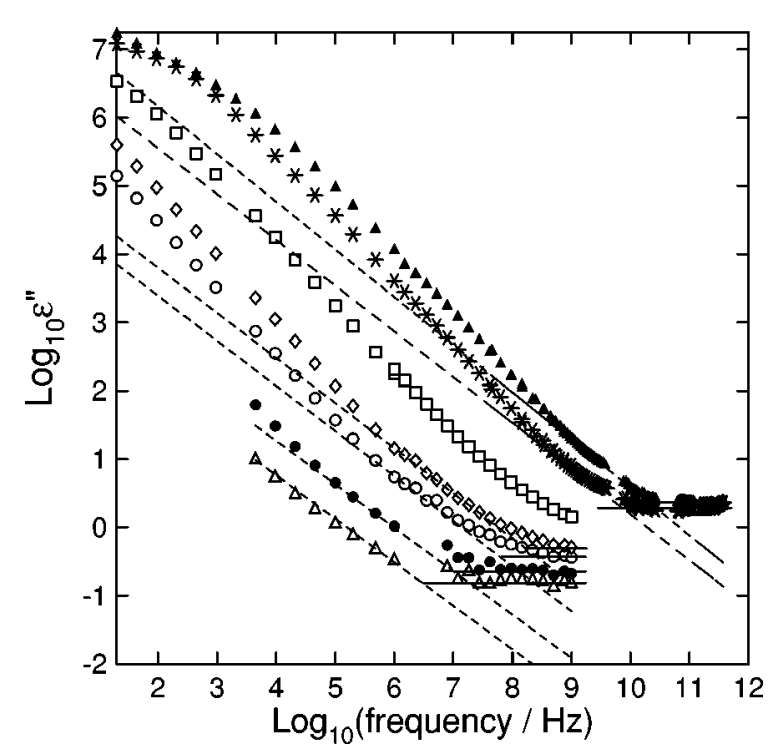

FIG. 3. Points are dielectric loss data of $\mathrm{CKN}$ by Lunkenheimer et al. at $T=350.3,356.4,365.4,370.0,385.0,412.0$, and $440.0 \mathrm{~K}$ (going from bottom to top). The dashed lines are the loss due to cooperative ion hopping calculated from the Kohlrausch fits to the electric modulus data and converted to a dielectric loss function of frequency. The crossover frequencies $\nu_{x 1}$ and $\nu_{x 2}$ (not shown) are determined from these data in the same manner as explained in Fig. 1. Errors in the data are smaller than the size of the symbols.

enough data points for such purpose. Errors of about half a decade wide can incur in determining $\nu_{x 1}$ and $\nu_{x 2}$ for some data considered in this work.

Having explained the procedure of determining the crossover frequencies by an example, we analyze in exactly the same manner the isothermal $\varepsilon^{\prime \prime}(\nu)$ data for thirteen temperatures shown in Figs. 3 and 4. All the data shown here are from Lunkenheimer et al. and some have not been published before in a refereed journal. For all temperatures, the dashed

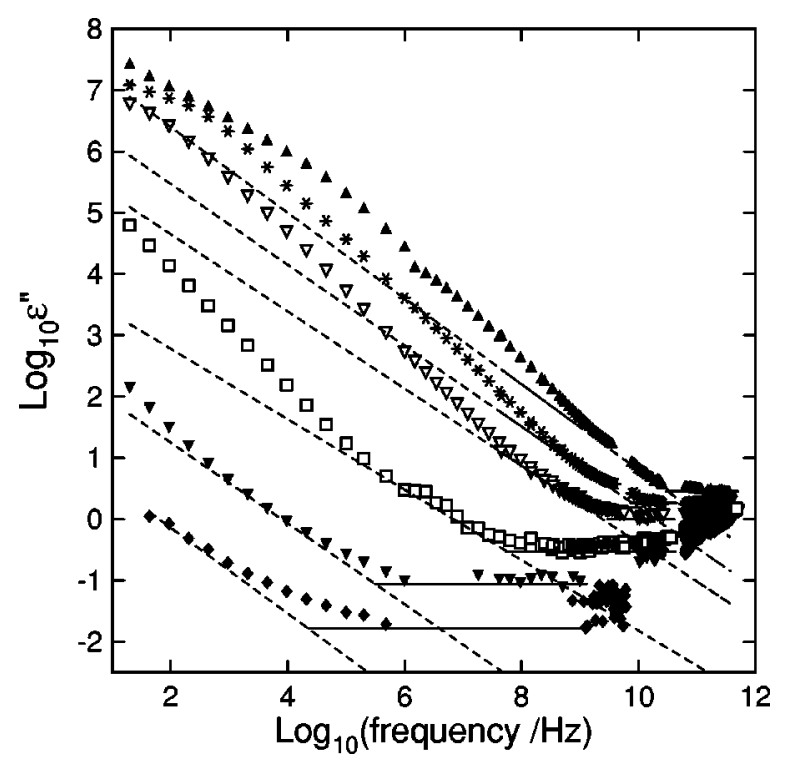

FIG. 4. Same as in Fig. 3 for temperatures of 325.0, 342.0, 361.0, 393.0, 417.0, and 468.0 K (from left to right). 


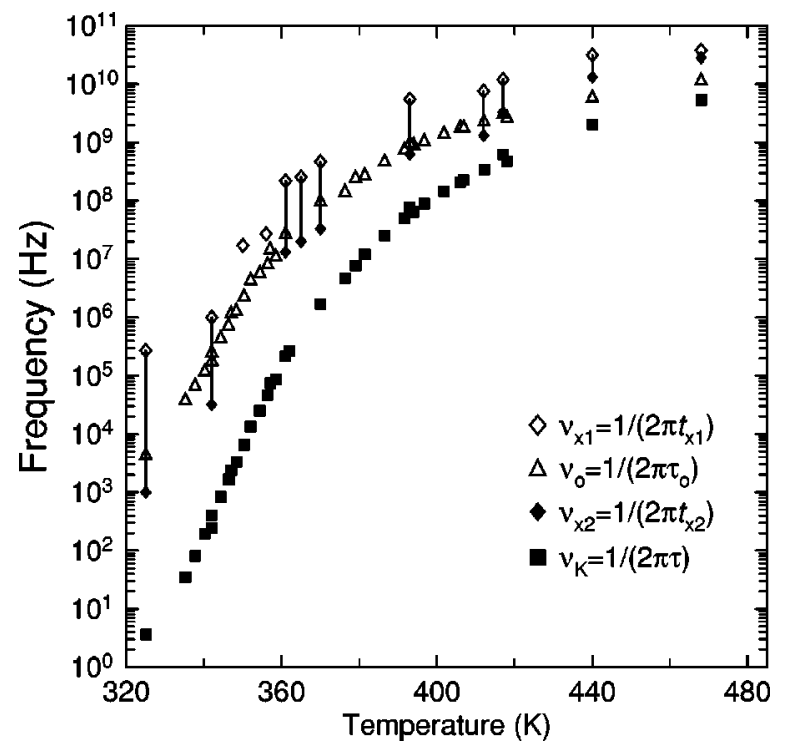

FIG. 5. Solid squares are the Kohlrausch conductivity relaxation frequency $\nu_{K} \equiv 1 /(2 \pi \tau)$, where $\tau$ is obtained from the fit to the data of $\mathrm{CKN}$ in the electric modulus representation. Open triangles are $\nu_{0} \equiv 1 /\left(2 \pi \tau_{0}\right)$, where $\tau_{0}$ is the independent relaxation time of the coupling model calculated from $\tau$ and $n$ obtained from the fit and $t_{c}=2 \mathrm{ps}$. The open and closed diamonds are the crossover frequencies $\nu_{x 1}$ and $\nu_{x 2}$ obtained from the procedure described in Figs. 1 and 2 .

line in these figures is the $\nu^{-\beta}$ dependence of $\varepsilon_{\beta}^{\prime \prime}(\nu)$ calculated from $M_{\beta}^{\prime \prime}$, the fit to $M^{\prime \prime}(\nu)$ data by Eqs. (4) and (5), and extrapolated to higher frequencies. The relaxation times $\tau(T)$ obtained from the fits are converted to a frequency $\nu_{K}$ $\equiv 1 /[2 \pi \tau(T)]$ shown in Fig. 5 as filled squares. In the same figure the crossover frequencies $\nu_{x 1} \equiv 1 /\left(2 \pi t_{x 1}\right)$ and $\nu_{x 2}$ $\equiv 1 /\left(2 \pi t_{x 2}\right)$ determined from the data in Figs. 3 and 4 are also shown (open diamonds for $\nu_{x 1}$ and filled diamonds for $\left.\nu_{x 2}\right)$. By definitions, $t_{x 1}$ and $t_{x 2}$ are naturally the crossover times. The crossover region is narrower at higher temperatures. Although broader at lower temperatures, the region is only about two and a half decades wide at $324 \mathrm{~K}$, the lowest measurement temperature below $T_{g}$. The other frequency, $\nu_{0} \equiv 1 /\left(2 \pi \tau_{0}\right)$, located between $\nu_{x 1}$ and $\nu_{x 2}$ in Figs. 1- 5, will be defined later.

\section{B. 0.80LiF-0.20Al $\left(\mathrm{PO}_{3}\right)_{3}$}

Electrical relaxation data at $293 \mathrm{~K}$ of a glassy ionic conductor, $0.80 \mathrm{LiF}-0.20 \mathrm{Al}\left(\mathrm{PO}_{3}\right)_{3}$, was obtained by Kulkarni et $a l .{ }^{31}$ over unusually broad frequency range. In Fig. 6, we show first their data as $\sigma^{\prime}$ as a function of frequency $\nu$. The existence of the NCL is exemplified by a near linear frequency dependence of the $\sigma^{\prime}$ data over about two decades, which terminates starting at $\nu_{x 1}$. The inset of the same figure shows the same data at low frequencies and up to $10^{6} \mathrm{~Hz}$ as $M^{\prime \prime}(\nu)$ to isolate the contribution from cooperative ion hopping. The line is the Kohlrausch fit by Eqs. (4) and (5) to the $M^{\prime \prime}(\nu)$ data with $\beta \equiv(1-n)=0.56$ and $\tau=8.7 \times 10^{-5} \mathrm{~s}$. At high frequencies the Kohlrasuch fit to $M^{\prime \prime}$ assume the $\nu^{-\beta}$ dependence and the corresponding $\sigma_{\beta}^{\prime}(\nu)$ assumes the $\nu^{1-\beta}$

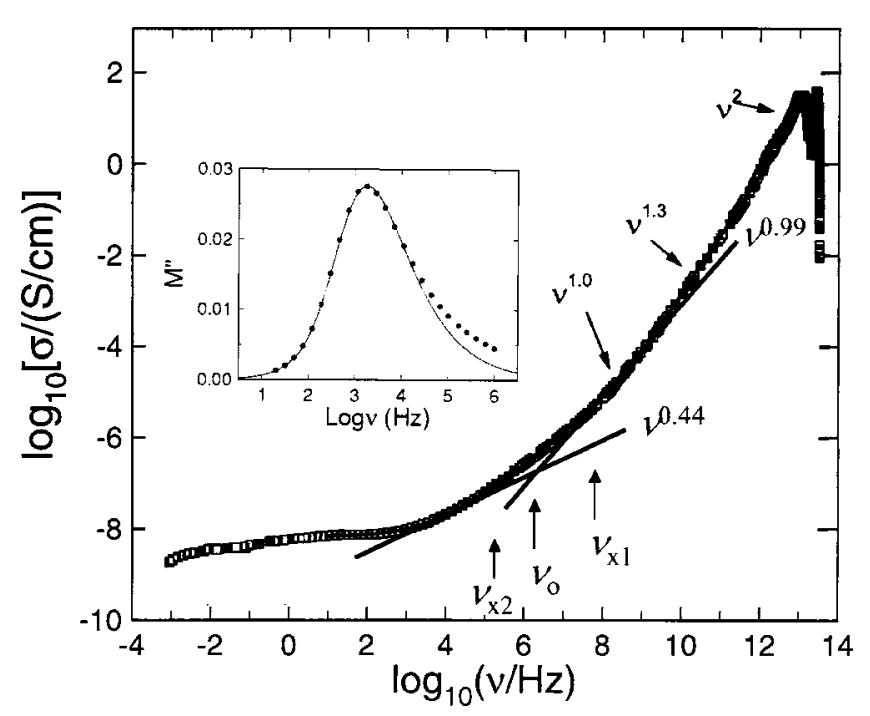

FIG. 6. $\log _{10} \sigma^{\prime}(\nu)$ versus $\log$ (frequency /Hz) plot of data at $T=293 \mathrm{~K}$ of $0.80 \mathrm{LiF}-0.20 \mathrm{Al}\left(\mathrm{PO}_{3}\right)_{3}$ obtained by Kulkarni et al. to show the near linear frequency dependence of the NCL by the line with slope equal to 0.99 . The line with slope 0.44 is the high frequency dependence of $\log _{10} \sigma^{\prime}(\nu)$ corresponding to the Kohlrausch fit to the electric modulus data (shown in the inset) with $n=0.44$. The dashed line in the main figure is the fully cooperative ion conductivity relaxation. The crossover frequencies $\nu_{x 1}$ and $\nu_{x 2}$ as well as the independent hopping frequency $\nu_{0}$ are determined from the data in the same manner as explained in Fig. 1.

dependence, which is shown by the straight line with slope 0.44 in Fig. 6. The $\sigma^{\prime}(\nu)$ data conform to the $\nu^{1-\beta}$ dependence of $\sigma_{\beta}^{\prime}(\nu)$ at frequencies below $\nu_{x 2}$. Thus, just like $\mathrm{CKN}$, the $\sigma^{\prime}(\nu)$ data of $0.80 \mathrm{LiF}-0.20 \mathrm{Al}\left(\mathrm{PO}_{3}\right)_{3}$ crossover from NCL to $\sigma_{\beta}^{\prime}(\nu)$ over the frequency range $\nu_{x 1}>\nu>\nu_{x 2}$.

\section{C. $0.5 \mathrm{Ag}_{2} \mathrm{~S}-0.5 \mathrm{GeS}_{2}$}

Figure 7 shows the data of glassy $0.5 \mathrm{Ag}_{2} \mathrm{~S}-0.5 \mathrm{GeS}_{2}$ from the work of Belin et al. at many temperatures. ${ }^{32,33}$ Only the data taken at 153 and $123 \mathrm{~K}$ are considered here because they show clearly the existence of the NCL over extensive frequency domains as indicated the solid (for $153 \mathrm{~K}$ ) and the dashed (for $123 \mathrm{~K}$ ) straight lines with $\nu^{1.0}$ dependence. The weak temperature dependence of the NCL is evident from this proximity of the two lines. The two vertical arrows pointing upwards indicate the location of $\nu_{x 1}$ for $153 \mathrm{~K}$ (at higher frequency) and $123 \mathrm{~K}$ (at lower frequency). Only $\nu_{x 2}$ can be determined from the data at $153 \mathrm{~K}$ and is indicated by another arrow labeled as such.

\section{D. $0.48(\mathrm{AgI})_{2}-\mathbf{0 . 5 2}_{2} \mathrm{Ag}_{2} \mathrm{SeO}_{4}$}

Conductivity relaxation data of $0.48(\mathrm{AgI})_{2}-0.52 \mathrm{Ag}_{2} \mathrm{SeO}_{4}$ at $153 \mathrm{~K}$ by Cramer and Buscher ${ }^{34}$ is shown in Fig. 8. The data in the intermediate frequency range is missing, but nevertheless the appearance of the NCL at high frequencies can be seen. The cooperative ion hopping at low frequencies is again characterized by the Kohlrausch fit to the electric modulus representation of the same data in the inset of Fig. 8 , with $\beta \equiv(1-n)=0.51$ and $\tau \equiv\left(1 / 2 \pi \nu_{K}\right)=3.4 \times 10^{-6} \mathrm{~s}$. 


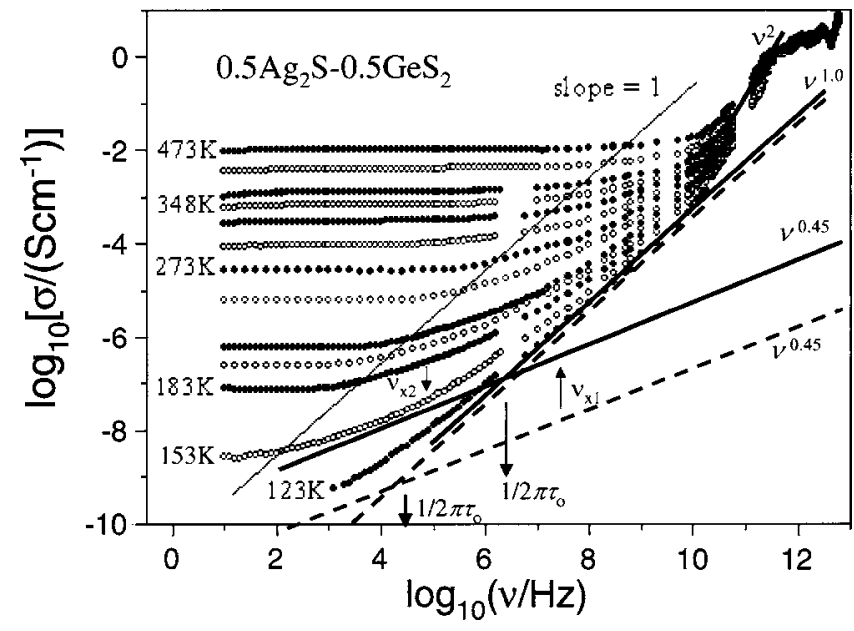

FIG. 7. Same as in Fig. 6 for glassy $0.5 \mathrm{Ag}_{2} \mathrm{~S}-0.5 \mathrm{GeS}_{2}$ from the work of Belin et al. The solid and dashed lines with slope one indicate the NCL for $T=153$ and $123 \mathrm{~K}$, respectively. The line with slope 0.45 is the high frequency dependence of $\log _{10} \sigma^{\prime}(\nu)$ corresponding to the Kohlrausch fit to the electric modulus data (not shown) with $n=0.45$. The crossover frequencies $\nu_{x 1}$ and $\nu_{x 2}$ as well as the independent hopping frequency $\nu \equiv 1 /\left(2 \pi \tau_{0}\right)$ are determined from the data at $153 \mathrm{~K}$ in the same manner as explained in Fig. 1. The arrow at the very bottom indicate the location of $\nu_{0}$ $\equiv 1 /\left(2 \pi \tau_{0}\right)$ for $T=123 \mathrm{~K}$.

Only $\nu_{x 2}$ can be determined from the data at $153 \mathrm{~K}$ and its location together with $\nu_{K}$ are indicated in Fig. 8.

\section{E. $0.44 \mathrm{LiBr}-0.56 \mathrm{Li}_{2} \mathrm{O}-\mathrm{B}_{2} \mathrm{O}_{3}$}

Conductivity data of $0.44 \mathrm{LiBr}-0.56 \mathrm{Li}_{2} \mathrm{O}-\mathrm{B}_{2} \mathrm{O}_{3}$ by Cramer et al. $^{35}$ at two temperatures are shown in Fig. 9. In this subsection we consider only the data at $323 \mathrm{~K}$, which exhibit the NCL at higher frequencies and the cooperative ion hopping contribution at lower frequencies. The asymptotic $\nu^{n}$ dependence of the Kohlrausch fit by Eqs. (4) and (5) to the lowerfrequency data with $n=0.48$ is shown in the figure. The er-

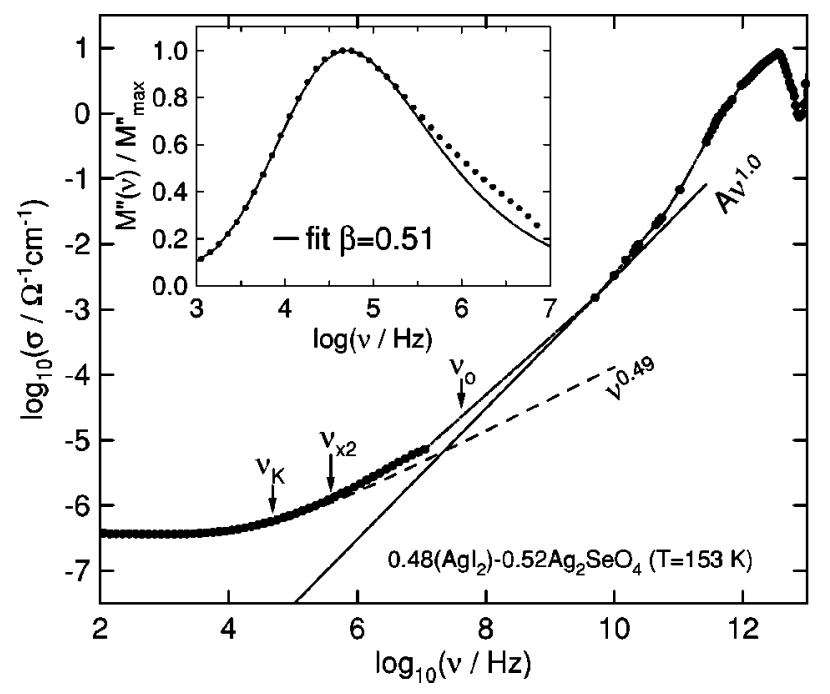

FIG. 8. Same as in Fig. 6 for glassy of $0.48(\mathrm{AgI})_{2}-0.52 \mathrm{Ag}_{2} \mathrm{SeO}_{4}$ at $T=153 \mathrm{~K}$ by Cramer and Buscher and $n=0.49$.

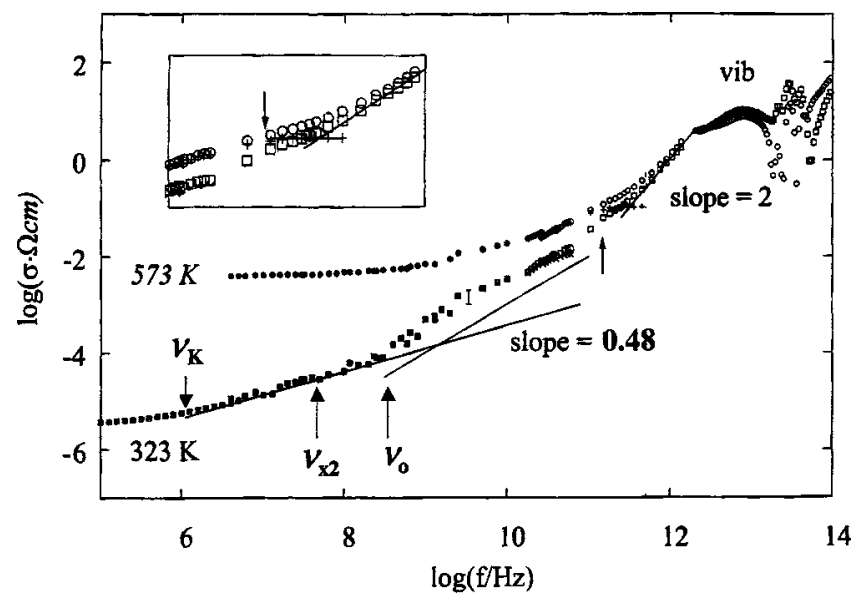

FIG. 9. The ac conductivity data of $0.44 \mathrm{LiBr}-0.56 \mathrm{Li} 20-\mathrm{B}_{2} \mathrm{O}_{3}$ by Cramer et al. at $T=573$ and $323 \mathrm{~K}$. Shown also is the high frequency vibrational contribution extending down to low frequencies with a $\nu^{2}$ dependence. Subtracting off this vibrational contribution from the data at $523 \mathrm{~K}$ leaves a frequency independent $\sigma_{0}(+)$, which corresponds to the independent ion hopping of all ions. The inset shows the same data in a small neighborhood of $10^{11} \mathrm{~Hz}$ and the data at the two temperatures in this region can be seen more clearly as well as for $T=573 \mathrm{~K}$ only the crossover of $\sigma^{\prime}(\nu)$ at a frequency $\nu_{c} \equiv 1 /\left(2 \pi t_{c}\right)$ of about $10{ }^{11} \mathrm{~Hz}$ (indicated by the vertical arrow at this frequency) from a $\nu^{n}$ dependence of fully cooperative hopping at high frequencies (line) to the frequency independent $\sigma_{0}$ (points denoted by + ). There is no intervening NCL at $573 \mathrm{~K}$. However at $T=323 \mathrm{~K}$, there is the NCL contribution with near $\nu^{1}$ dependence to $\sigma^{\prime}(\nu)$ at high frequencies (steeper line with slope equal to one) and the crossover to the $\nu^{n}$ dependence of fully cooperative hopping at high frequencies (line with slope $n=0.48$ ). The quality of the data in the NCL regime is not good enough to determine $\nu_{x 1}$, but $\nu_{x 2}, \nu_{K}$, and $\nu_{0}$ have been determined in the manner as in Fig. 6 for $323 \mathrm{~K}$ and are indicated.

rors of the data are sufficiently large that preempt determination of $\nu_{x 1}$ and only the approximate location of $\nu_{x 2}$ is indicated.

\section{F. $\mathrm{Ag}_{7} \mathrm{GeSe}_{5} \mathrm{I}$}

The conductivity data of the crystalline ionic conductor $\mathrm{Ag}_{7} \mathrm{GeSe}_{5} \mathrm{I}$ at the lowest temperature of $114 \mathrm{~K}$ shown in Fig. 10 exhibit the NCL at high frequencies (dashed line with slope equal to 1) and the crossover to the cooperative hopping conductivity. ${ }^{36}$ The asymptotic $\nu^{n}$ dependence of the Kohlrausch behavior is shown by another dashed line. The locations of the crossover frequencies $\nu_{x 1}$ and $\nu_{x 2}$ are indicated by arrows.

\section{G. $x \mathrm{Na}_{2} \mathrm{OO}(1-x)\left[0.04 \mathrm{~B}_{2} \mathrm{O}_{3}-0.96 \mathrm{SiO}_{2}\right]$ with $x=0.00044$}

The Vycor glass $x \mathrm{Na}_{2} \mathrm{OO}(1-x)\left[0.04 \mathrm{~B}_{2} \mathrm{O}_{3}-0.96 \mathrm{SiO}_{2}\right]$ with $x=0.00044$ contains very few $\mathrm{Na}^{+}$ions. ${ }^{15,37}$. Nevertheless, its conductivity relaxation data measured at $313^{\circ} \mathrm{C}$ show the existence of the NCL at higher frequencies and the crossover to a near exponential ion hopping with a small $n$ $=0.05$. These features together with the locations of the crossover frequencies $\nu_{x 1}$ and $\nu_{x 2}$, are indicated by arrows in Fig. 11. 


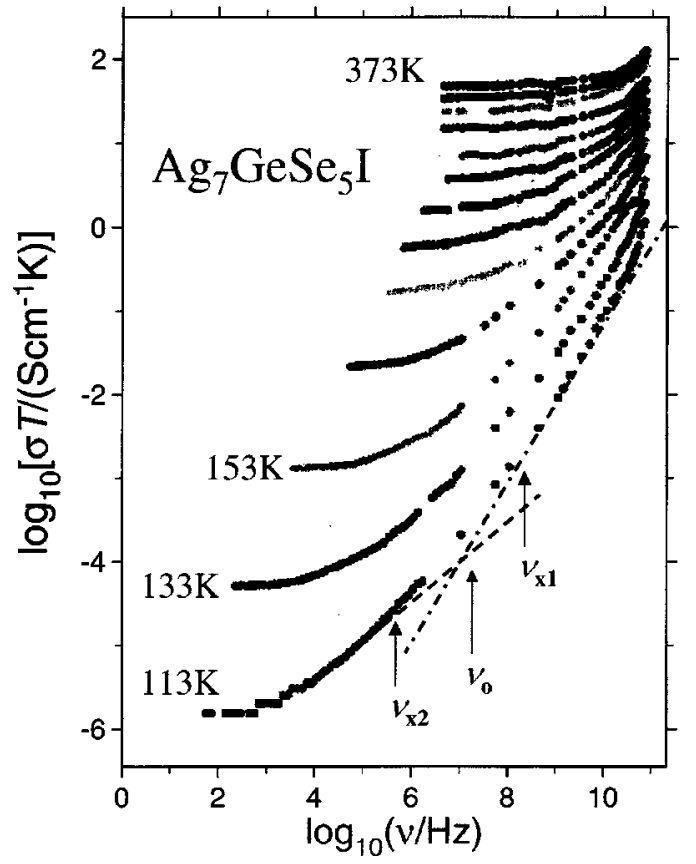

FIG. 10. Same as in Fig. 6 for the crystalline ionic conductor $\mathrm{Ag}_{7} \mathrm{GeSe}_{5}$ I. Data after Pradel and Ribes (unpublished). At the lowest temperature of $113 \mathrm{~K}, \nu_{x 1}, \nu_{x 2}$, and $\nu_{0}$ have been determined in the manner as in Fig. 6 and are indicated.

\section{H. $x \mathrm{~K}_{2} \mathrm{O}-(1-x) \mathrm{GeO}_{2}$ glasses with $x=0.02$ and 0.0023}

A similar situation as the Vycor glass is found in the conductivity relaxation data of two $x \mathrm{~K}_{2} \mathrm{O}-(1-x) \mathrm{GeO}_{2}$ glasses with $x=0.02$ and 0.0023 obtained by Jain et al. ${ }^{16}$ They have characterized the cooperative ion hopping by the Kohlrausch fit to the electric modulus data with $\beta \equiv(1-n)=0.89$ and 0.93 , respectively, for $x=0.02$ and 0.0023 . Here we show

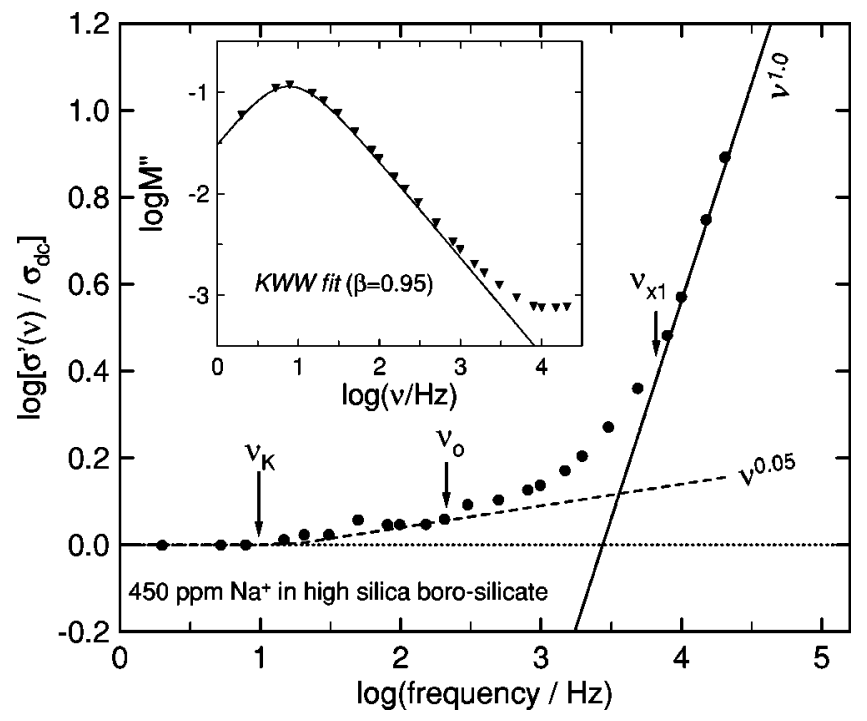

FIG. 11. Same as in Fig. 6 for a Vycor glass $x \mathrm{Na}_{2} \mathrm{O}(1-x)$ $\times\left[0.04 \mathrm{~B}_{2} \mathrm{O}_{3}-0.96 \mathrm{SiO}_{2}\right]$ with $x=0.00044$ at $T=313{ }^{\circ} \mathrm{C}$. Data after Simmons et al. Here $n$ is equal to 0.05 . The locations of the crossover frequencies $\nu_{x 1}$ and $\nu_{x 2}$, as well as $\nu_{K}$ and $\nu_{0}$ are indicated by the vertical arrows.

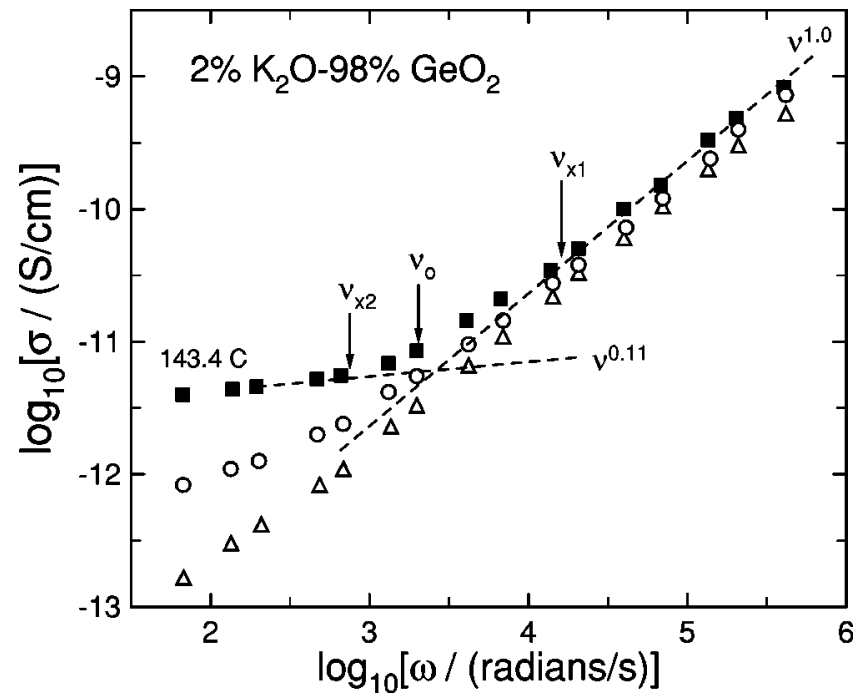

FIG. 12. Same as in Fig. 11 for $x \mathrm{~K}_{2} \mathrm{O}-(1-x) \mathrm{GeO}_{2}$ glasses with $x=0.02$. Data after Jain et al. Here $n$ is equal to 0.11 obtained by Jain et al. from fit to the electric modulus (not shown). The locations of the crossover frequencies $\nu_{x 1}$ and $\nu_{x 2}$, as well as $\nu_{0}$ are indicated by the vertical arrows.

only the data of $x=0.02$ in Fig. 12. In the figure, for the data at $143.4{ }^{\circ} \mathrm{C}$, we drew two lines corresponding to the two power laws $\nu^{1.0}$ and $\nu^{0.11}$ (thick solid lines) and from these lines and the experimental data we determine the two crossover frequencies.

\section{THE INDEPENDENT ION HOPPING RELAXATION TIME $\tau_{0}$ FROM THE COUPLING MODEL}

The coupling model (CM) for ionic conductors ${ }^{26-28}$ considers only the case that all the mobile ions have high probability of hopping out of their cages. All of them are ready to hop to neighboring sites with the relaxation rate $\tau_{0}^{-1}$ except their interactions and correlations make the independent hops of each ion simultaneously impossible. The result is a slowed-down cooperative motion of all the ions with dynamics that is heterogeneous and the correlation function is changed at some time $t_{c}$ from the linear exponential

$$
\Phi(t)=\exp \left(-t / \tau_{0}\right)
$$

for independent hops to the stretched exponential function $\exp \left[-(t / \tau)^{1-n}\right]$ [Eq. (5)]. The relation between $\tau$ and $\tau_{0}$ is given by

$$
\tau=\left[t_{c}^{-n} \tau_{0}\right]^{1 /(1-n)}
$$

with $t_{c}=2$ ps determined previously by high frequency measurements for ionic conductors. ${ }^{34,35,38-40}$ Experimental evidences of the existence of such a change of dynamics in a neighborhood of $t_{c}$ are found in high frequency and highest temperature $\sigma(\omega)$ measurements of molten CKN, glassy $0.44 \mathrm{LiBr}-0.56 \mathrm{Li}_{2} 0-\mathrm{B}_{2} \mathrm{O}_{3}$ and other glasses by Cramer et al. , $^{34,35,38,39}$ after the $\omega^{2}$-dependent vibrational absorption contribution is removed from the data. The temperature must be high enough such that there is no intervening NCL, and 
TABLE I. Activation enthalpies $E_{a}$ of $0.5 \mathrm{AgI}-0.5 \mathrm{AgPO}_{3}$ (Refs. 46,47) and $\mathrm{Ag}_{2} \mathrm{~S}-\mathrm{GeS}_{2}$ (Ref. 48) from short time ionic diffusion (neutron time of flight) measurements. The Kohlrausch exponent $\beta$ and the activation enthalpy $E_{\sigma}$ of conductivity relaxation observed in the same glasses (Refs. 41,42) at lower temperatures and frequencies in the fully cooperative hopping region are also included. The near equality between $E_{a}$ and $\beta E_{\sigma}$ is found, consistent with the change to independent relaxation at times shorter than $t_{c} \approx 2 \mathrm{ps}$ and probed by quasielastic neutron scattering.

\begin{tabular}{lcccc}
\hline \hline Glass & $E_{\sigma}$ & $\beta$ & $\beta E_{\sigma}$ & $E_{a}$ \\
\hline $\mathrm{AgI}^{-} \mathrm{AgPO}_{3}$ & $21 \mathrm{~kJ} / \mathrm{mol}$ & 0.44 & $9.2 \mathrm{~kJ} / \mathrm{mol}$ & $8.7 \mathrm{~kJ} / \mathrm{mol}$ \\
$\mathrm{Ag}_{2} \mathrm{~S}_{-}-\mathrm{GeS}_{2}$ & $0.34 \mathrm{eV}$ & 0.45 & $0.153 \mathrm{eV}$ & $0.15 \mathrm{eV}$ \\
\hline \hline
\end{tabular}

the cooperative ion hopping term (having at high frequencies the $\nu^{n}$ dependence for the ac conductivity) crosses over directly to the independent ion hopping term (having a frequency independent conductivity). An example can be seen in Fig. 9, and more clearly in the inset. At the highest measurement temperature $573 \mathrm{~K}$ in $0.44 \mathrm{LiBr}-0.56 \mathrm{Li}_{2} 0-\mathrm{B}_{2} \mathrm{O}_{3}$, there is no intervening NCL and therefore after subtracting out the vibrational contribution, the result should be solely from ion diffusion. The result levels off rather abruptly at about $10^{11} \mathrm{~Hz}$ (or a time of about $2 \mathrm{ps}$ ) to a plateau, i.e., a frequency independent value. Now a frequency independent conductivity corresponds to an exponential correlation function, Eq. (7). Thus the change from independent hopping [Eq. (7)] to cooperative hopping [Eq. (5)] with decreasing frequency at about $10^{11} \mathrm{~Hz}$ or a time $t_{c} \approx 1$ to $2 \mathrm{ps}$ is evident from the figure. Similar changes were found in crystalline ionic conductors $\mathrm{Na} \beta$ alumina ${ }^{41,42}$ and $\mathrm{RbAg}_{4} \mathrm{I}_{5} .{ }^{43}$

Other evidences of the change of dynamics near $T_{c} \approx 1$ to 2 ps were found from the change of the temperature dependence of the dc conductivity of many ionic conductors, ${ }^{44,45}$ when it exceeds about $1 \mathrm{~S} / \mathrm{cm}$. Another support comes from quasielastic neutron scattering (time of flight) measurements at short times (in the picosecond and sub picoseconds range) of the ionic diffusion coefficient as a function of temperature in superionic glasses. Such measurements of ion diffusion at short times in glassy ionic conductors $\mathrm{AgI}-\mathrm{AgPO}_{3}$ and $0.5 \mathrm{Ag}_{2} \mathrm{~S}-0.5 \mathrm{GeS}_{2}$ were made by two groups. ${ }^{46-48}$ The glass $0.5 \mathrm{Ag}_{2} \mathrm{~S}-0.5 \mathrm{GeS}_{2}$ was the same one discussed earlier in Sec. IIC. From the quasielastic neutron scattering data it was found that the activation enthalpy of the short-time diffusion coefficient $E_{a}$ is smaller than $E_{\sigma}$ of the dc conductivity, ${ }^{48}$ and approximately equal to $(1-n) E_{\sigma} \cdot{ }^{41,42}$ The results are shown here in Table I. Here, $(1-n)$ is the Kohlrausch exponent of the fully cooperative hopping in Eq. (5) and $E_{\sigma}$ is the activation enthalpy of the fully cooperative hopping relaxation time $\tau$ in Eq. (5) or the dc conductivity at much lower temperatures and longer times than those in quasielastic neutron scattering experiments. It is easy to verify from Eq. (8) that the product $(1-n) E_{\sigma}$, is the activation enthalpy of the independent hops relaxation time $\tau_{0}$. Thus, quasielastic neutron scattering measurements in the picosecond and subpicoseconds range found that the ions are hopping independently in this short time regime, indicating that $t_{c}$ is of the order of $1 \mathrm{ps}$.
The independent relaxation time $\tau_{0}(T)$ of the CM can be calculated by Eq. (8) from the experimental values of $\tau$ and $(1-n)$, obtained from fitting $M^{*}(\omega)$ data by Eq. (2), and using $t_{c}=2 \mathrm{ps}$. The interpretation of $\tau_{0}(T)$ given by the CM is the relaxation time for the ion vibrating in its potential well to come out by overcoming the energy barrier $E_{a}$ by thermal activation. If $\tau$ has Arrhenius temperature dependence and $n$ is constant over a temperature region, then it follows from Eq. (8) that $\tau_{0}(T)$ should also have Arrhenius temperature dependence $\tau_{0}(T)=\left[t_{c}^{n} \tau^{1-n}\right]=\tau_{\infty} \exp \left(E_{a} / k T\right)$ over the same temperature range. From its simple nature of $\tau_{0}$, the reciprocal of its prefactor $\tau_{\infty}$ must be identifiable with the vibration angular frequency of the ion inside the well. An example that indicates $\tau_{0}$ is indeed the thermally activated relaxation time of an ion to hop out of its potential well is the $\mathrm{CM}$ analysis of conductivity relaxation and hyper Raman scattering data of yttria stabilized zirconia. ${ }^{45}$

All previous applications of the CM dealt with the cooperative ion hopping in the long time regime. It is important to emphasize that the dynamics in the early time regime, when most of the mobile ions are still caged, have not been included in all previous applications. At sufficiently short times/low temperatures, few ions have succeeded to move away from their original sites, although the number increases with time albeit very slowly. A near constant loss (NCL) then originates from very slow decay of the correlation function of the cage with time, which ultimately is due to those few ions that have succeeded to hop out of their cages to neighboring sites over decades of time in this short time regime. Naturally these successful hops are executed by independent ion jumps with rate $\tau_{0}^{-1}$ because the successful hops are rare and there is absence of cooperativity. Only for times short compared with $\tau_{0}$, or equivalently for frequencies high compared with $\nu_{0} \equiv 1 / 2 \pi \tau_{0}$, the successful hops out the cages will be few and infrequent. Thus the NCL exists only at times much shorter than $\tau_{0}$ or frequencies much higher than $\nu_{0}$. This point will be revisited after a comparison of $\nu_{0}$ with $\nu_{x 1}$ and $\nu_{x 2}$ of CKN has been made.

\section{RELATION OF $\nu_{0}$ WITH $\nu_{x 1}$ AND $\nu_{x 2}$}

The ion conductivity relaxation data of CKN in Figs. 1-4 were analyzed by Eq. (2) and the two parameters $\tau(T)$ and $[1-n(T)]$ had been determined by Lunkenheimer et al. ${ }^{29-30}$ The data cover an extended temperature range from below and above the glass transition temperature $T_{g}$. As typical of relaxation of glass-forming substances above $T_{g}$, the relaxation frequency $\nu_{K} \equiv 1 / 2 \pi \tau$ is not Arrhenius and the Kohlrausch parameter $\beta \equiv[1-n(T)]$ is temperature dependent. From these parameters, the independent ion relaxation time $\tau_{0}(T)$ had also been calculated previously by Lunkenheimer ${ }^{29,30}$ with $t_{c}=2$ ps determined experimentally for $\mathrm{CKN}{ }^{38,39}$ The independent ion hopping frequency $\nu_{0}$ $\equiv 1 / 2 \pi \tau_{0}$, is plotted against temperature in Fig. 5. At high temperatures where $\nu_{x 1}$ and $\nu_{x 2}$ are close to each other, $\nu_{0}(T)$ lies close to these two frequencies. At lower temperatures, where the crossover region $\nu_{x 1}>\nu>\nu_{x 2}$ becomes broader, it is remarkable that $\nu_{0}$ invariably falls inside it. The same is found also for $0.4 \mathrm{Ca}\left(\mathrm{NO}_{3}\right)_{2}-0.6 \mathrm{RbNO}_{3}(\mathrm{CRN})$ from 
similar analysis given to the electrical relaxation data obtained by Lunkenheimer et al. ${ }^{29,30}$

In a similar manner as described for $\mathrm{CKN}$ in the preceding paragraph, we have determined $\nu_{0}$ for the other glassy and crystalline ionic conductors considered in Sec. II B-II H. When comparing $\nu_{0}$ with $\nu_{x 1}$ and $\nu_{x 2}$ in Figs. 6- 12, we find again in these other ionic conductors that $\nu_{x 1}>\nu_{0}>\nu_{x 2}$, as in the molten salt CKN. The presently found relation between $\nu_{0}$ and crossover frequencies $\nu_{x 1}$ and $\nu_{x 2}$ for glassy and molten CKN ionic conductors and other glassy and crystalline ionic conductors may not be an accident because they are independently and separately determined quantities. First, $\nu_{0}$ is determined from the low frequency dispersion and the use of the coupling model relation, Eq. (8). Secondly, $\nu_{x 1}$ and $\nu_{x 2}$ are determined separately by the terminations of two loss mechanisms, NCL and cooperative hopping [Eqs. (4) and (5)], that have very different temperature dependences. We may recall that NCL has weak temperature dependence approximately described by $\exp \left(T / T_{0}\right),{ }^{18}$ while the frequency dependent loss from ion hopping has a strong thermally activated temperature dependence.

\section{NCL FROM VERY SLOWLY DECAYING CAGE DYNAMICS}

The main thrust of the present work is to bring out from experimental data properties of the crossover from the NCL to fully cooperative ion hopping conductivity. It is not intended to give here a rigorous theory for the origin of the NCL, the part that is unrelated to ADWP or similar explanations. Nevertheless it is worthwhile to give some theoretical ideas of the origin of the NCL that are consistent with the empirical results found in this work and a previous work. ${ }^{18}$

\section{A. Cage decay}

For a fixed cage with a potential that does not change with time, there is no NCL that extends over decades of time and increases in extent with decreasing temperature similar to that found in ionic conductors. In a harmonic well, the mean square displacement of the ion given by the solution of Chandrasekhar ${ }^{49}$ increases rapidly with time to a maximum value that is proportional to $T$. In general, the cage or the potential-well that confines a mobile ion is determined by the matrix atoms and the other mobile ions. This fact is evident from the form of typical potentials used in molecular dynamics simulations. ${ }^{50,51}$ The cage is not permanent and it changes or decays because of at least two reasons. First, the spatial relation with the immobile matrix atoms can change with time as suggested by the broadening of the first peak of the van Hove function with time. ${ }^{51}$ Second, other ions may be leaving their cages and the probability of them doing so increases with time as can be seen from the development of intensity at neighboring initially vacant sites of the van Hove function with time. The contribution to cage decay from the second cause was established by the study of the motion of concentrated colloidal particles suspension using confocal microscopy by Weeks and Weitz. ${ }^{52}$ In this case, the cage is formed entirely by neighboring particles of the same kind as the caged particle. On the other hand, in ionic conductors there are also the immobile matrix atoms that form the cage. By defining particles as nearest neighbors if their separation is less than a cutoff distance set by the first minimum of the pair correlation function, Weeks and Weitz define a cage correlation function $C_{\text {cage }}(\Delta t)$, by the fraction of particles with the same neighbors at time $T$ and at $t+\Delta t$, after averaging over all $T$. Deduced from the confocal microscopy data, $C_{\text {cage }}(\Delta t)$ shows decay, which becomes slower as the volume fraction of the particles increases and their diffusion rate decreases. This loss is observed as an increase of the mean square displacement $\left\langle r^{2}\right\rangle$ of the ion with time, in response to the decay of the cage. If the cage decay is a sufficiently slow function of time, the corresponding increase of $\left\langle r^{2}\right\rangle$ is also very slow. In fact, we observe from the data of $\left\langle r^{2}\right\rangle$ reported by Weeks and Weitz for volume fraction $\phi$ equal to 0.52 and 0.56 as a function of time, that $\left\langle r^{2}\right\rangle$ increases very slowly with time at shorter times when the cage decays also very slowly. $\left\langle r^{2}\right\rangle$ increases approximately as $c t^{\alpha}$ with $\alpha \approx 0.10$ in the time regime of roughly between 5 to $50 \mathrm{~s}$ for $\phi$ equal to 0.52 and 5 to $100 \mathrm{~s}$ for $\phi$ equal to 0.56 , where the cage decay function exhibits also very slow decay.

Such a slow increase of $\left\langle r^{2}\right\rangle$ with time is equivalent to a NCL if the particle is charged similar to ions in ionic conductors, and the quantity measured is dielectric loss due to conductivity relaxation. This result can be seen from the following approximate (neglecting cross correlations and that the Haven ratio is not unity) relation between mean square displacement and complex conductivity

$$
\sigma^{*}(\omega)=-\omega^{2} \frac{N q^{2}}{6 k T} \int_{0}^{\infty}\left\langle r^{2}(t)\right\rangle \exp (-i \omega t) d t
$$

where $N$ is the density of mobile ions, $q$ the ion charge, $k$ the Boltzmann constant, and $T$ the temperature. For $\left\langle r^{2}\right\rangle \propto c t^{\alpha}$, Eq. (9) gives $\sigma^{\prime}(\omega) \propto \omega^{1-\alpha}$ and $\varepsilon^{\prime \prime}(\omega) \propto \omega^{-\alpha}$ and therefore a NCL if $\alpha$ is small. If the mean square displacement would increase logarithmically with time as $\left\langle r^{2}\right\rangle \propto \log t$, the dielectric loss $\varepsilon^{\prime \prime}(\omega)$ would be practically flat, and $\sigma^{\prime}(\omega) \propto \omega^{1.0}$. As with the colloidal system, the cages confining ions in ionic conductors also decay with time because at any time there is nonzero probability of ions jumping out to a neighboring site.

\section{B. Independent hops as cause of decay of cage correlation function}

At sufficiently short times, when most of the ions are still being caged, the successful jumps out of the cages are independent of each other because there are very few such events and therefore no cooperative dynamics are involved. The independent ion jump has rate $1 / \tau_{0}$. At times much shorter than $\tau_{0}$, successful hops of ions out of their cages are rare and few in between. Hence the decay of the cages or the increase of $\left\langle r^{2}\right\rangle$ with time has to be very slow, resulting in the NCL. Naturally the NCL defined as such is a very slow relaxation process and can exist in the time regime $t \ll \tau_{0}$, where the increase of the number of successful hops of ions out of their cages over decades of time is insignificant. The 
NCL terminates at some time $t_{x 1} \equiv 1 / 2 \pi \nu_{x 1}$, when afterwards the successive independent ion hops have become more significant and the loss can no longer be considered as nearly constant as a function of $\ln t$, i.e., there is a more rapid increase of $\left\langle r^{2}\right\rangle$ with time beyond $t_{x 1}$, which can no longer justify the NCL to continue. This process is dynamically heterogeneous. Still $t_{x 1}$ has to be much smaller than $\tau_{0}$ (or $\nu_{x 1} \gg \nu_{0}$ ) because $\tau_{0}$ is the characteristic time for the ions to leave their cages. The number of ions successful hopping out of the cages continues to increase beyond $t_{x 1}$, changes in $\left\langle r^{2}\right\rangle$ become more rapid leading to increasing deviation from the near NCL. However, only at times $t$ sufficiently longer than $\tau_{0}$, i.e., for $t>t_{x 2}>\tau_{0}$, will all ions have almost certain probability of successfully hopping out their cages, but then their mutual interactions make all such independent hops impossible. This is the start of the fully cooperative ion hopping regime described by the Kohlrausch stretched exponential function, Eq. (5) and by definition $t_{x 2} \equiv 1 / 2 \pi \nu_{x 2}$, is the onset time. Hence, from $t_{x 2}>\tau_{0}$, it follows that $\nu_{x 2}<\nu_{0}$. When the fully cooperative hopping regime is entered, the dependences, $\left\langle r^{2}\right\rangle \propto t^{1-n}, \varepsilon_{\beta}^{\prime \prime}(\nu) \propto \nu^{-1+n}$ and $\sigma_{\beta}^{\prime}(\nu) \propto \nu^{n}$, are observed. These power laws are just the shorter time $(t \ll \tau)$ or high frequency $\left(\nu>\nu_{K}\right)$ parts of these quantities for the fully cooperative hopping with the Kohlrausch correlation function given by Eq. (5), the longer time $(t \gg \tau)$ parts of which are $\left\langle r^{2}\right\rangle \propto t, \varepsilon_{\beta}^{\prime \prime}(\nu) \propto \nu^{-1}$ and $\sigma_{\beta}^{\prime}(\nu)=\sigma_{\mathrm{dc}}$.

From the qualitative theory given above, we have a qualitative explanation of the origin of the NCL, and the time regime $t<t_{x 1}$ or frequency regime $\nu>\nu_{x 1}$ where it can exist. We also have given physical meaning to the crossover times (frequencies) $t_{x 1}\left(\nu_{x 1}\right)$ and $t_{x 2}\left(\nu_{x 2}\right)$. The qualitative theory also explains the empirical results we found from the collection of data presented in this work. These empirical results include (1) the relation $t_{x 1} \ll \tau_{0}\left(\nu_{x 1} \gg \nu_{0}\right)$ and (2) the fact that $\tau_{0}$ always lies inside the crossover region, $t_{x 1}<t<t_{x 2}$ or $\nu_{0}$ always lies in between $\nu_{x 1}$ and $\nu_{x 2}$.

The coupling model embodied by Eqs. (5), (7), and (8) for fully cooperative ion hopping at longer times $\left(t>t_{x 2}\right)$ enables the independent relaxation time $\tau_{0}$ to be calculated from the low frequency $\left(\nu<\nu_{x 2}\right)$ experimental data. Thus the empirical results found show that the coupling model (usually applied to the fully cooperative hopping regime) also has an impact on the NCL and the broad crossover from the NCL to the fully cooperative hopping regime. The impact is the ability of $\tau_{0}$ to relate to the time $t_{x 1}$ or frequency $\nu_{x 1}$ when the NCL is terminated, and to locate the time scale of the broad crossover from the NCL to the fully cooperative hopping.

\section{TEMPERATURE DEPENDENCE OF THE NCL}

We have proposed that the NCL exists in the short time (high frequency) regime, $t_{\mathrm{on}}<t<t_{x 1}\left(\nu_{\mathrm{on}}>\nu>\nu_{x 1}\right)$ where there are few thermally activated independent jumps of the ions out of their cages with the relaxation rate $2 \pi \nu_{0} \equiv 1 / \tau_{0}$ $=\left(1 / \tau_{\infty}\right) \exp \left(-E_{a} / k T\right)$. Here $t_{\text {on }}\left(\nu_{\text {on }}\right)$ is the onset time (frequency) of the NCL. From experimental data that show the NCL extending to very high frequencies, $t_{\mathrm{on}}$ is of the order of $10^{-11}$ to $10^{-12} \mathrm{~s}$. The value of $t_{x 1}$ depends on the choice of the exponent $\alpha \approx 0$ used to define the NCL and is the time beyond which the experimental data $\sigma^{\prime}(\omega)$ departs from the $A \omega^{1-\alpha}$ dependence. But, whatever the choice of $\alpha \approx 0, t_{x 1}$ has to be much less than $\tau_{0}$ in order to satisfy the condition for generation of the NCL that there are few successful independent ion jumps out of their cages throughout the period $t_{\text {on }}<t<t_{x 1}$. Therefore $\exp \left(-t_{x 1} / \tau_{0}\right)$ is still close to unity and its value is determined by the choice of the exponent $\alpha$ of the NCL. The value of $\exp \left(-t_{x 1} / \tau_{0}\right)$ is fixed for all temperatures by the convention used to define that the NCL terminates at $t_{x 1}$. Hence $t_{x 1}$ has the same thermally activated temperature dependence as $\tau_{0}$, and it is written out explicitly here as $t_{x 1}(T)=t_{\infty} \exp \left(E_{a} / k T\right)$. We have considered here the case of glassy ionic conductors, which have Arrhenius temperature dependence for $\tau_{0}$. For molten ionic conductors such as CKN, $\tau_{0}$ is not Arrhenius and hence also $t_{x 1}(T)$, as shown in Fig. 5.

Again, from the definition of $t_{x 1}$, the mean square displacement $\left\langle r^{2}\right\rangle_{\mathrm{NCL}}$ corresponding to the NCL increases by the same small amount in the period, $t_{\mathrm{on}}<t<t_{x 1}(T)$, at all temperatures. However, because $t_{x 1}$ is thermally activated, this same increase of $\left\langle r^{2}\right\rangle_{\mathrm{NCL}}$ is spread over a number of decades of time given by $\left[\log _{e}\left(t_{x 1}\right)-\log _{e}\left(t_{\mathrm{on}}\right)\right] / 2.303$. Therefore the mean square displacement is inversely proportional to $\log _{e}\left(t_{x 1} / t_{\mathrm{on}}\right)$. From this and the relation between conductivity and mean square displacement [Eq. (9)], we deduce that the intensity of the NCL measured by $A$ in Eqs. (1) and (2) is given by the proportionality relation

$$
A \propto \frac{1}{E_{a}}\left[1-\left(k T / E_{a}\right) \log _{e}\left(t_{\mathrm{on}} / t_{\infty}\right)\right]^{-1} .
$$

This expression is well approximated by

$$
A \propto \exp \left(T / T_{0}\right)
$$

at $T \ll T_{0}$, with

$$
T_{0} \approx E_{a} / k \log _{e}\left(t_{\mathrm{on}} / t_{\infty}\right) .
$$

$T_{0}$ is a positive number because $\ln \left(t_{\mathrm{on}} / t_{\infty}\right)$ is a positive number from the fact that $t_{x 1}<\tau_{0}, t_{\infty}$ is even shorter than the prefactor of $\tau_{0}, \tau_{\infty}$, which is the reciprocal of a vibrational attempt frequency. Thus the weak temperature dependence of the NCL is captured by our interpretation of the origin of the NCL. Since the result given by Eqs. (10) and (11) are obtained from a qualitative theory, we do not expect that it will accurately describe the temperature dependence of the NCL. Particularly if considered over a very extended temperature range where another source of contribution to NCL may come into play at very low temperatures and high frequencies. Note also that we have not taken into account the contribution to the measured $\left\langle r^{2}\right\rangle$ from the local vibrational dynamics, which has its own temperature dependence similar to that of the Debye-Waller factor. The latter will enhance the temperature dependence of the NCL over and above that given by Eq. (10).

We note from Eq. (10) that at constant temperature $A$ should decrease with increasing $E_{a}$, had other factors that determine the absolute value of $A$ be the same for all ionic 
conductors. If this condition holds for many ionic conductors, then an approximate anti-correlation between $A$ and $E_{a}$ may exist at constant $T$.

\section{CONCLUSION}

Empirically we found from the rich experimental conductivity relaxation spectra of CKN over a wide frequency and temperature range and other glassy and crystalline ionic conductors that the NCL existing at short times (high frequencies) terminates at $t_{x 1}\left(\nu_{x 1}\right)$. The fully cooperative ion hopping contribution takes over at a later time (lower frequency) $t_{x 2}\left(\nu_{x 2}\right)$. The crossover from the NCL to the fully cooperative ion hopping therefore occurs over a broad time (frequency) range $t_{x 1}<t<t_{x 2}\left(\nu_{x 1}>\nu>\nu_{x 2}\right)$. The width of this range in CKN is no more than two and a half decades wide, and it decreases monotonically with increasing temperature. The same is found in several glassy ionic conductors and in a crystalline ionic conductor. The width of the crossover region seems a bit larger in glasses having smaller stretch exponent $1-n$ of the correlation function for the fully cooperative ion hopping, such as $0.48(\mathrm{AgI})_{2}-0.52 \mathrm{Ag}_{2} \mathrm{SeO}_{4}$.

We have calculated from the parameters of the stretched exponential correlation function fit to the data in the fully cooperative ion hopping regime, the independent ion hopping relaxation time $\tau_{0}$ of the coupling model. Most fascinating is the finding that $t_{x 1} \ll \tau_{0}$ and $\tau_{0}$ always lies within the crossover region $t_{x 1}<t<t_{x 2}$. These findings help us to propose that the NCL originates from very slow decay of the cages in the short time regime where most of the ions are still caged. There are few and infrequent successful hops of ions to new sites and the mean square displacement increases very slowly with the logarithm of time. This condition for the appearance of NCL is satisfied at times $t<t_{x 1}$ and $t_{x 1}$ $\ll \tau_{0}$, such that $\exp \left(-t / \tau_{0}\right)$ decreases very slowly with $\ln (t)$. At times longer than $\tau_{0}$ and starting at some later time $t_{x 2}$, all ions have almost certain probability of hopping out of their cages to new sites. However, the hops of all ions cannot be successful now for a different reason, which is the interaction and correlation between the ions. The only option available is the fully cooperative hopping of all ions with dynamics that is heterogeneous and the averaged hopping rate is slowed down compared with the independent relaxation rate $1 / \tau_{0}$. The correlation function of the fully cooperative hopping is given by Eq. (5) and its relaxation time $\tau$ is related to $\tau_{0}$ by Eq. (8) according to the coupling model. Naturally the dynamics of ions in the crossover region, particularly at the long times, is interpreted as the gradual build up of cooperative motion with increasing time because more ions can successfully hop out of their cages. Fully cooperative hopping is established when $t_{x 2}$ is reached.

The confocal microscopy data of colloidal particles systems obtained by Weeks and Weitz are cited to provide direct experimental support of the proposed origin of NCL from the very slow decay of the cages. This observation in colloidal systems, as well as in ionic conductors presented in this work, indicates the proposed mechanism for NCL is possibly very general, applicable to other systems such as glass formers without mobile ions. ${ }^{53-55}$

We point out that there are also other contributions that can give rise to NCL different from our proposed mechanism and may be relevant in data obtained under different conditions (such as at low temperatures and high frequencies) than that for the data considered in this work. One source that have been discussed by many workers comes from relaxation of a distribution of asymmetric double well potentials (ADWP), which seem to have been seen in glasses without mobile ions, in CKN as well as in glassy ionic conductors. In the model of Gilroy-Phillips, ${ }^{56}$ the ADWP contribute a loss with $\omega^{a}$ dependence with $a$ not constant but increasing linearly with temperature. Thus the ADWP contribution possibly can be the NCL observed only at low temperatures. The ADWP contribution to susceptibility $\chi^{\prime \prime}(\omega)$ of CKN and other glass formers by dynamic light scattering experiments showed that it exists at very high frequencies above $1 \mathrm{GHz}$ at lower temperatures, but is not a NCL according to our definition, because the power $a$ indeed increases with temperature to reach significantly large values. ${ }^{57-59}$ The dielectric relaxation data of CKN within the same temperature range taken over a much wider frequency range down to much lower frequencies show the NCL occurs in a frequency range at much lower frequencies than $1 \mathrm{GHz}$. Thus, it seems that there is an ADWP contribution that cannot be identified with $\mathrm{NCL}$ at higher frequencies than $1 \mathrm{GHz}$ at least in this temperature range. However, there is also a NCL at lower frequencies in the same temperature range. The dielectric data collected so far are not good enough in the frequency range above $1 \mathrm{GHz}$ and at temperatures below $T_{g}$ to be able to make a quantitative comparison with the dynamic light scattering data. Nevertheless, the limited data suggest possible good correspondence with the light scattering data and hence the observation also of the ADWP contribution by dielectric relaxation in this high frequency range while the NCL of present interest appears in the lower frequencies. But at higher temperature the observed fast relaxation in the same frequency regime can no longer be explained by the ADWP in the model by Gilroy and Phillips. Experimentally, from the light scattering susceptibility spectra, the power $a$ starts to decrease with increasing temperature and the spectrum tends to become the NCL. ${ }^{57}$ This is consistent with the dielectric data, which shows that at these higher temperatures, there is a NCL in the $\mathrm{GHz}$ range.

\section{ACKNOWLEDGMENTS}

We thank P. Lunkenheimer, A. Kulkarni, A. Pradel, C. Cramer, and $\mathrm{H}$. Jain for sending their experimental data. The work performed at NRL was supported by ONR. C. León is supported in Spain by CICYT Grant No. MAT2001-3713C04. 
${ }^{1}$ G.C. Garton, Discuss. Faraday Soc. 42, 161 (1946).

${ }^{2}$ A.E. Owen, Prog. Ceram. Sci. 3, 77 (1963).

${ }^{3}$ J.M. Stevels, in Hamdbuch der Physik, edited by S. Flugge (Springer, Berlin, 1957), Vol. 20, p. 372.

${ }^{4}$ J. Wong and C.A. Angell, Glass Structure by Spectroscopy (Dekker, New York, 1976).

${ }^{5}$ W.K. Lee, J.F. Liu, and A.S. Nowick, Phys. Rev. Lett. 67, 1559 (1991).

${ }^{6}$ X. Lu and H. Jain, J. Phys. Chem. Solids 55, 1433 (1994).

${ }^{7}$ C. Cramer, K. Funke, and T. Saatkamp, Philos. Mag. B 71, 701 (1995).

${ }^{8}$ D.L. Sidebottom, P.F. Green, and R.K. Brow, Phys. Rev. Lett. 74, 5068 (1995).

${ }^{9}$ H. Jain and X. Lu, J. Non-Cryst. Solids 196, 285 (1996).

${ }^{10}$ C. León, M.L. Lucia, and J. Santamaria, Phys. Rev. B 55, 882 (1997).

${ }^{11}$ K.L. Ngai, H. Jain and O. Kanert, J. Non-Cryst. Solids 222, 383 (1997).

${ }^{12}$ A.S. Nowick, A.V. Vaysleb, and W. Liu, Solid State Ionics 105, 121 (1998).

${ }^{13}$ K.L. Ngai, J. Non-Cryst. Solids 248, 194 (1999).

${ }^{14}$ K.L. Ngai, J. Chem. Phys. 110, 10576 (1999).

${ }^{15}$ K.L. Ngai and C.T. Moynihan, Mater. Res. Bull. 23, 51 (1998).

${ }^{16}$ H. Jain and S. Krishnaswami, Solid State Ionics 105, 129 (1998).

${ }^{17}$ A.S. Nowick, B.S. Lim, and A.V. Vaysleb, J. Non-Cryst. Solids 172-174, 1243 (1994).

${ }^{18}$ C. León, A. Rivera, A. Várez, J. Sanz, J. Santamaria, and K.L. Ngai, Phys. Rev. Lett. 86, 1279 (2001).

${ }^{19}$ A. Rivera, C. León, C.P.E. Varsamis, G.D. Chryssikos, K.L. Ngai, C.M. Roland, and L.J. Buckley, Phys. Rev. Lett. 88, 125902 (2002).

${ }^{20}$ A.K. Jonscher, Dielectric Relaxation in Solids (Chelsea Dielectric Press, London, 1983).

${ }^{21}$ For a review of various representation of conductivity relaxation data see C.T. Moynihan, J. Non-Cryst. Solids 172-174, 1395 (1994); 203, 359 (1996); Solid State Ionics 105, 75 (1998).

${ }^{22}$ U. Strom, K.L. Ngai, and O. Kanert, J. Non-Cryst. Solids 131133, 1011 (1991).

${ }^{23}$ C. León, J. Santamaria, M.A. Paris, J. Sanz, J. Ibarra, and L.M. Torres, Phys. Rev. B 56, 5302 (1997).

${ }^{24}$ C. León, M.L. Lucia, J. Santamaria, and F. Sánchez-Quesada, Phys. Rev. B 57, 41 (1998).

${ }^{25}$ A. Rivera, C. León, J. Sanz, J. Santamaria, C.T. Moynihan, and K.L. Ngai, Phys. Rev. B 65, 224302 (2002).

${ }^{26}$ K.L. Ngai, Comments Solid State Phys. 9, 121 (1979).

${ }^{27}$ K.L. Ngai, R.W. Rendell, and H. Jain, Phys. Rev. B 30, 2133 (1984).

${ }^{28}$ K.Y. Tsang and K.L. Ngai, Phys. Rev. E 56, R17 (1997).

${ }^{29}$ P. Lunkenheimer, A. Pimenov, and A. Loidl, Phys. Rev. Lett. 78, 2995 (1997).

${ }^{30}$ P. Lunkenheimer, Dielectric Spectroscopy of Glassy Dynamics (Shaker, Aachen, 1999).

${ }^{31}$ A. Kulkarni, P. Lunkenheimer, and A. Loidl, Ceram. Trans. 92, 115 (1999).
${ }^{32}$ R. Belin, G. Taillades, A. Pradel, and M. Ribes, Solid State Ionics 136-137, 1025 (2000).

${ }^{33}$ M. Ribes, E. Bychkov, and A. Pradel, J. Optoelectron. Adv. Mater. 3, 665 (2001).

${ }^{34}$ C. Cramer and M. Buscher, Solid State Ionics 105, 109 (1998).

${ }^{35}$ C. Cramer, K. Funke, M. Buscher, and A. Happe, Philos. Mag. B 71, 713 (1995).

${ }^{36}$ A. Pradel and M. Ribes (unpublished).

${ }^{37}$ J.H. Simmons, P.B. Elterman, C.J. Simmons, and R.K. Mohr, J. Am. Ceram. Soc. 62, 158 (1979).

${ }^{38}$ C. Cramer, K. Funke, and T. Saatkamp, Philos. Mag. 71, 701 (1995).

${ }^{39}$ K.L. Ngai, C. Cramer, T. Saatkamp, and K. Funke, in NonEquilibrium Phenomena in Supercooled Fluids, Glasses and Amorphous Materials, edited by M. Giordano, D. Leporini, and M.P. Tosi (World Scientific, Singapore, 1996), p. 3.

${ }^{40}$ K.L. Ngai, J. Non-Cryst. Solids 248, 194 (1999).

${ }^{41}$ K.L. Ngai, J. Phys. (Paris), Colloq. 2, 61 (1992).

${ }^{42}$ K.L. Ngai and O. Kanert, Solid State Ionics 53-55, 936 (1992).

${ }^{43}$ K. Funke, Prog. Solid State Chem. 22, 111 (1993).

${ }^{44}$ K.L. Ngai, G.N. Greaves, and C.T. Moynihan, Phys. Rev. Lett. 80, 1018 (1998).

${ }^{45}$ K.L. Ngai, Philos. Mag. 77, 187 (1998).

${ }^{46}$ M. Tachez, R. Mercier, J.P. Malugani, and A.J. Dianoux, Solid State Ionics 20, 93 (1986).

${ }^{47}$ A.J. Dianoux, M. Tachez, R. Mercier, and J.P. Malugani, J. NonCryst. Solids 131-133, 973 (1991).

${ }^{48}$ A.P. Owens, A. Pradel, M. Ribes, and S.R. Elliott, J. Non-Cryst. Solids 131-133, 1104 (1991); in Solid State Ionics II, edited by G.-A. Nazri et al., MRS Symposia Proceedings No. 210 (Materials Research Society, Pittsburgh, 1991), p. 621.

${ }^{49}$ S. Chandrasekhar, Rev. Mod. Phys. 15, 1 (1943).

${ }^{50}$ C. Huang and A.N. Cormack, J. Chem. Phys. 95, 3634 (1991).

${ }^{51}$ J. Habasaki and Y. Hiwatari, Phys. Rev. E 59, 6962 (1999).

${ }^{52}$ E.R. Weeks and D.A. Weitz, URL http://arxiv. org/abs/cond-mat/ 0107279

${ }^{53}$ A. Hoffmann, F. Kremer, E.W. Fischer, and A. Schönhals in $\mathrm{Su}$ percooled Liquids, Advances and Novel Applications, edited by J.T. Fourkas et al., ACS Symposium Series No. 676 (American Chemical Society, Washington, DC, 1997), p. 309.

${ }^{54}$ J. Wiedersich, T. Blochowicz, S. Benkhof, A. Kudlik, N.V. Surovtsev, C. Tschirwitz, V.N. Novikov, and E. Rössler, J. Phys.: Condens. Matter 11, A147 (1999).

${ }^{55}$ R. Casalini and K.L. Ngai, J. Non-Cryst. Solids 293, 318 (2001).

${ }^{56}$ K.S. Gilroy and W.A. Phillips, Philos. Mag. B 43, 735 (1981).

${ }^{57}$ J. Gapinski, W. Steffen, A. Patkowski, A.P. Sokolov, A. Kisliuk, U. Buchenau, M. Russina, F. Mezei, and H. Schober, J. Chem. Phys. 110, 2312 (1999).

${ }^{58}$ J. Wiedersich, N.V. Surovtsev, and E. Rössler, J. Chem. Phys. 113, 1143 (2000).

${ }^{59}$ J. Wiedersich, N.V. Surovtsev, V.N. Novikov, E. Rössler, and A.P. Sokolov, Phys. Rev. B 64, 064207 (2001). 